\title{
Nonequilibrium transport through a disordered molecular nanowire
}

\author{
P. Thiessen, ${ }^{1}$ E. Díaz, ${ }^{1, *}$ R. A. Römer, ${ }^{2}$ and F. Domínguez-Adame ${ }^{1,2}$ \\ ${ }^{1}$ GISC, Departamento de Física de Materiales, Universidad Complutense, E-28040 Madrid, Spain \\ ${ }^{2}$ Department of Physics and Centre for Scientific Computing, University of Warwick, Coventry, CV4 7AL, United Kingdom \\ (Received 22 December 2016; revised manuscript received 3 May 2017; published 30 May 2017)
}

\begin{abstract}
We investigate the nonequilibrium transport properties of a disordered molecular nanowire. The nanowire is regarded as a quasi-one-dimensional organic crystal composed of self-assembled molecules. One orbital and a single random energy are assigned to each molecule while the intermolecular coupling does not fluctuate. Consequently, electronic states are expected to be spatially localized. We consider the regime of strong localization, namely, the localization length is smaller than the length of the molecular wire. Electron-vibron interaction, taking place at each single molecule, is also considered. We investigate the interplay between static disorder and electron-vibron interaction in response to either an applied electric bias or a temperature gradient. To this end, we calculate the electric and heat currents when the nanowire is connected to leads, using the Keldysh nonequilibrium Green's function formalism. At intermediate temperature, scattering by disorder dominates both charge and heat transport. We find that the electron-vibron interaction enhances the effect of the disorder on the transport properties due to the decrease of the coherent electron tunneling among molecules.
\end{abstract}

DOI: 10.1103/PhysRevB.95.195431

\section{INTRODUCTION}

Anderson localization of the electronic wave function in a random medium is a major paradigm of quantum coherence in condensed matter physics: noninteracting electrons in three dimensions are spatially localized for sufficiently large disorder [1] and in one-dimension all the states of random systems become exponentially localized for any amount of disorder due to coherent backscattering [2]. With a few exceptions [3-9], a single-parameter scaling theory [10] generally provides a very accurate picture of the electronic states in noninteracting disordered systems. In real solids, however, electrons interact with each other and with lattice vibrations, and these interactions may affect the transport properties of disordered systems. For instance, electron-phonon interaction can decrease the ability of electrons to form localized states and hence increase charge mobility $[11,12]$.

The advent of nanotechnology has renewed attention on Anderson localization because it is enhanced in lowdimensional systems [10]. Among the large variety of materials with technological interest in this field, crystalline molecular systems are gaining relevance as active components in electronic nanodevices [13]. Unfortunately, the detailed mechanisms of charge transport in molecular systems driven out of equilibrium are still controversial, posing a complicated scenario for the theoretical description of experiments [14]. For example, it has been argued that, depending on the various energy scales involved (electron bandwidth, zero-point energy of molecular vibrations, thermal energy), electron-phonon coupling may not play a significant role on charge transport even at room temperature, as deduced from inelastic electron tunneling spectroscopy experiments [15-18]. When the charge carriers interact with low-energy intermolecular modes, they move in a slowly changing potential landscape that gives rise to the so-called transient localization [19]. On the other hand, intramolecular modes occur at high frequency due to the

\footnotetext{
*elenadg@ fis.ucm.es
}

stretching of stiff covalent bonds. Coupling to those modes may strongly alter charge transport [20] and even lead to the self-trapping of charge carriers, provided that the relaxation energy (the energy gained upon the deformation of lattice around the carrier) largely exceeds the kinetic energy gained from the carrier tunneling to neighbor molecules [19].

In this work, we aim at exploring the intermediate regime when the electron-vibron interaction is not strong and the effect on the transport properties of a long and pristine molecular nanowire (MNW) are expected to be small. We are particularly interested in the interplay between static disorder and polaronic effects on the charge and heat transport properties in this regime. The MNW will be regarded as a quasi-onedimensional organic crystal of self-assembled molecules [21]. Specifically, we consider a MNW with the two ends connected to ideal leads and assume that the electrons interact with a vibrational degree of freedom localized at each molecule. Disorder in the electronic environment of each molecule can originate from interactions with a random environment of solute molecules and ions surrounding the MNW. Unlike Ref. [22], we propose a two-probe configuration to diminish the conductance fluctuations found in four-probe setups. It should be stressed that we neglect the lateral motion of electrons in the MNW. Grange has recently established that the current-voltage characteristics show a transition from wide to narrow wires, displaying additional peaks due to resonances with optical phonons [23]. Since we only deal with quasi-one-dimensional MNWs, this transition is beyond the scope of our work. We will use the Keldysh nonequilibrium Green's function formalism [24] to obtain the spectral function as well as the electric and heat currents through the MNW, driven out of equilibrium by either an applied electric bias or temperature gradient.

One of our main findings is the strong effect of disorder on the electron transport properties of the MNW when the electron-vibron interaction is taken into account. The enhancement of the localization effects can be traced back to the so-called exponential suppression of tunneling [25]. This amounts to reducing the coherent electron tunneling among neighbor molecules when the electron-vibron interaction is 


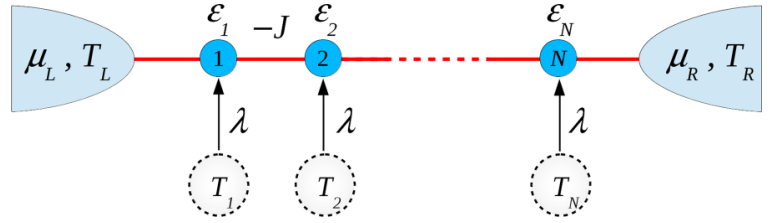

FIG. 1. Schematic diagram of the MNW with intermolecular hopping parameter $J$. The MNW is also connected to left and right leads. We take into account an intramolecular electron-vibron interaction, $\lambda$ being the coupling constant and $T_{i}$ the temperature of the bath for each molecule. The $\varepsilon_{i}$ denote the local molecular energies and $\mu_{L, R}$ and $T_{L, R}$ are the chemical potentials and the temperatures in the left and right leads, respectively.

non-negligible. Consequently, the ratio between the magnitude of disorder, defined as the width of the distribution of site energies, and the electron hopping parameter among neighbor molecules becomes larger for higher temperatures. This can actually be viewed as an effective increase of the disorder. Notice that, due to the zero-point motion, the hopping parameter is already significantly decreased at $T=0$, leading to a stronger effective disorder even in the absence of background temperature.

\section{MODEL AND FORMALISM}

We consider a MNW composed of $N$ self-assembled molecules and connected to left $(L)$ and right $(R)$ leads by tunneling couplings, as shown schematically in Fig. 1. The chemical potentials of the leads under a bias voltage $V$ are given by $\mu_{L}=\mu+e V / 2$ and $\mu_{R}=\mu-e V / 2$, where $\mu$ is the equilibrium chemical potential and $e$ the electron charge. The lead temperatures are set as $T_{L}=T-\Delta T / 2$ and $T_{R}=T+\Delta T / 2$, where $T$ is a background temperature and $\Delta T$ is the temperature difference between the hot and the cold leads.

Only one energy level in each molecule is assumed relevant and electron-electron interaction is neglected. On-site energies are subjected to disorder representing inhomogeneous broadening. Then, the energy level of the molecule $i$ splits as $\varepsilon_{i}=\varepsilon+\Delta \varepsilon_{i}$, where $\Delta \varepsilon_{i}$ is a random uncorrelated variable whose distribution function is $P\left(\Delta \varepsilon_{i}\right)=1 / W$ if $\left|\Delta \varepsilon_{i}\right|<W / 2$ and zero otherwise. $W$ will be referred to as magnitude of disorder. In addition, the electron interacts with a local vibration mode at each molecule which we assume of the same frequency $\omega_{0}$ for simplicity. Besides the different temperature of the leads, we introduce a temperature gradient in the system by setting a different temperature $T_{i}$ of each bath (see Fig. 1). In this work, we interpolate linearly $T_{i}$ between $T_{L}$ and $T_{R}$.

\section{A. The coupled electron-vibron system}

The Hamiltonian describing the whole system splits into three contributions as $H=H_{0}+H_{\mathrm{e}-\text { leads }}+H_{\mathrm{e}-\text { vib }}$ [26]. The term $H_{0}$ describes the dynamics of the noninteracting system (we set $\hbar=1$ )

$$
\begin{aligned}
H_{0}= & \sum_{i=1}^{N} \varepsilon_{i} c_{i}^{\dagger} c_{i}-J \sum_{i=1}^{N-1}\left(c_{i}^{\dagger} c_{i+1}+c_{i+1}^{\dagger} c_{i}\right) \\
& +\omega_{0} \sum_{i=1}^{N} a_{i}^{\dagger} a_{i}+\sum_{\alpha k} \varepsilon_{\alpha k} d_{\alpha k}^{\dagger} d_{\alpha k} .
\end{aligned}
$$

Here, $d_{\alpha k}^{\dagger}\left(d_{\alpha k}\right)$ denotes the creation (annihilation) operator of a conduction electron in the lead $\alpha=L, R$ with crystal momentum $k$ and energy $\varepsilon_{\alpha k}$. Similarly, $c_{i}^{\dagger}\left(c_{i}\right)$ is the creation (annihilation) operator of an electron in the molecule $i$ with energy $\varepsilon_{i} . J$ indicates the bare intermolecular hopping energy and is assumed constant and positive. It is worth noticing that $J$ depends on the particular distance and orientation between neighboring units and it could change due to molecular vibrations [27]. However, this issue is beyond our current study and further works should consider it. Finally, the creation (annihilation) operator of a vibron in the molecule $i$ with frequency $\omega_{0}$ is denoted by $a_{i}^{\dagger}\left(a_{i}\right)$.

The MNW is tunnel-coupled to both leads, as shown schematically in Fig 1. Therefore the corresponding Hamiltonian reads

$$
H_{\mathrm{e}-\mathrm{leads}}=\sum_{\alpha k i}\left(V_{\alpha k i} d_{\alpha k}^{\dagger} c_{i}+V_{\alpha k i}^{*} c_{i}^{\dagger} d_{\alpha k}\right) .
$$

Self-trapping has been commonly formulated within the framework of the small polaron theory based on a local Holstein-type coupling [28] between the carrier and the intramolecular modes. Quanta of the intramolecular vibrations are usually referred to as vibrons. The Holstein-type coupling between the electron and the vibrons [28] can be written as

$$
H_{\mathrm{e}-\mathrm{vib}}=\lambda \sum_{i}\left(a_{i}^{\dagger}+a_{i}\right) c_{i}^{\dagger} c_{i} .
$$

The electron-vibron coupling constant $\lambda$ is assumed uniform over the MNW. We now apply the polaron (Lang-Firsov [29]) nonperturbative canonical transformation when the coupling to the leads is not strong $\left(\left|V_{\alpha k i}\right|<\lambda\right)$. In such a case, it is reasonable to replace the displacement operator $X_{i}=$ $\exp \left[-\left(\lambda / \omega_{0}\right)\left(a_{i}^{\dagger}-a_{i}\right)\right]$ that emerges after the transformation by its thermal expectation value evaluated in equilibrium $\left\langle X_{i}\right\rangle$. Notice that such polaron transformation and the subsequent replacement turns the original many-body problem into an effective one-body problem (see Appendix A for details). The transformed Hamiltonian is approximately given by

$$
\begin{aligned}
\widetilde{H} \approx & \sum_{i=1}^{N} \widetilde{\varepsilon}_{i} c_{i}^{\dagger} c_{i}-\sum_{i=1}^{N-1}\left(\widetilde{J}_{i} c_{i}^{\dagger} c_{i+1}+\text { H.c. }\right)+\sum_{i=1}^{N} \omega_{0} a_{i}^{\dagger} a_{i} \\
& +\sum_{\alpha k i}\left(\widetilde{V}_{\alpha k i} d_{\alpha k}^{\dagger} c_{i}+\text { H.c. }\right)
\end{aligned}
$$

with $\widetilde{\varepsilon}_{i}=\varepsilon_{i}-\lambda^{2} / \omega_{0}$ being the renormalized energy level of the molecule $i, \widetilde{J}_{i}=J \exp \left[-\xi_{i}\left(T_{i}\right) / 2-\xi_{i+1}\left(T_{i+1}\right) / 2\right]$ and $\widetilde{V}_{\alpha k i}=V_{\alpha k i} \exp \left[-\xi_{i}\left(T_{i}\right) / 2\right]$. Here, H.c. stands for Hermitian conjugate. We have defined $\xi_{i}\left(T_{i}\right)=g\left(2 n_{i}+1\right), n_{i}=$ $1 /\left[\exp \left(\omega_{0} / k_{B} T_{i}\right)-1\right]$, and $g=\lambda^{2} / \omega_{0}^{2}$. Notice that the higher the temperature, the smaller the dressed couplings among the molecules $\widetilde{J}_{i}$ and between them and the leads $\widetilde{V}_{\alpha k i}$, as pictured 


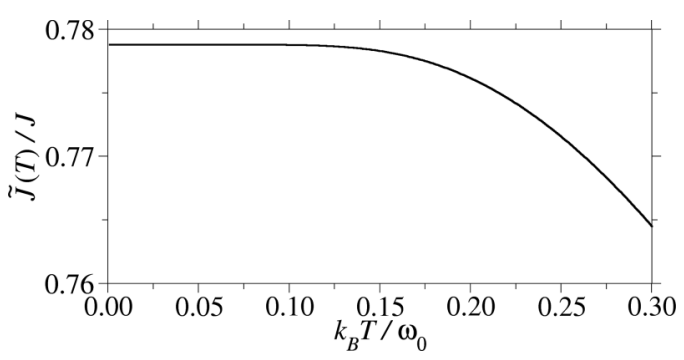

FIG. 2. Temperature dependence of the dressed hopping energy $\widetilde{J}$ among the molecules at an electron-vibron coupling strength of $\lambda=0.25$, in units of the bare hopping energy $J$. In the absence of temperature gradient, the dressed hopping energy is independent of the site index. The observed monotonic decay continues for larger $T$ values.

in Fig. 2. This will be referred to as exponential suppression of tunneling [25]. It is worth mentioning that $\widetilde{J}_{i} / J<1$ at $T=0$ due to the zero-point motion.

\section{B. Nonequilibrium transport properties}

Nonequilibrium transport properties of an interaction region coupled to two leads can be obtained with the help of the Keldysh nonequilibrium Green's function technique [30]. This procedure is detailed in Appendix B.

After transformation into Fourier space, the calculation of the greater and lesser Green's functions, $G^{>}(\omega)$ and $G^{<}(\omega)$, allows us to obtain the spectral matrix

$$
\mathcal{A}(\omega)=\mathrm{i}\left[G^{>}(\omega)-G^{<}(\omega)\right],
$$

along with the spectral function $A(\omega)=\operatorname{Tr}[\mathcal{A}(\omega)] / N$. In addition, we can also calculate the symmetrized electric current [24]

$$
\begin{aligned}
J_{e}= & \frac{e}{2} \int \frac{d \omega}{2 \pi} \operatorname{Tr}\left[\left(\Gamma^{L}-\Gamma^{R}\right) \mathrm{i} G^{<}(\omega)\right. \\
& \left.+\left(f_{L}(\omega) \Gamma^{L}-f_{R}(\omega) \Gamma^{R}\right) \mathcal{A}(\omega)\right] .
\end{aligned}
$$

Here, $f_{\alpha}(\omega)=1 /\left\{\exp \left[\left(\omega-\mu_{\alpha}\right) / k_{B} T_{\alpha}\right]+1\right\}$ is the FermiDirac distribution function of the lead $\alpha$. The matrices which encode the coupling to the leads, $\Gamma^{\alpha}$, are taken symmetric with $\Gamma_{1,1}^{L}=\Gamma_{N, N}^{R}\left(\Gamma_{i j}^{\alpha}=0\right.$ otherwise $)$. Notice that we will neglect their $k$ dependence by relying on the wide-band limit approximation and take these matrices as energy-independent magnitudes.

Unlike the electric current, the heat current is not necessarily conserved due to the coupling to the heat baths and Joule heating. We will mainly concentrate on the heat current from the left lead to the MNW,

$$
J_{Q}^{L}=\int \frac{d \omega}{2 \pi} \omega \operatorname{Tr}\left[\Gamma^{L} \mathrm{i} G^{<}(\omega)+f_{L}(\omega) \Gamma^{L} \mathcal{A}(\omega)\right]-\mu_{L} \frac{J_{e}}{e},
$$

which is expected to differ from the heat current $J_{Q}^{R}$ from the right lead to the MNW. For instance, due to the symmetry of the system at $\Delta T=0$, the heat current $J_{O}^{L}(-\Delta T)$ from the left lead to the system at $-\Delta T$ is equal to the energy flux $J_{Q}^{R}(\Delta T)$ from the right lead to the system at $\Delta T$.

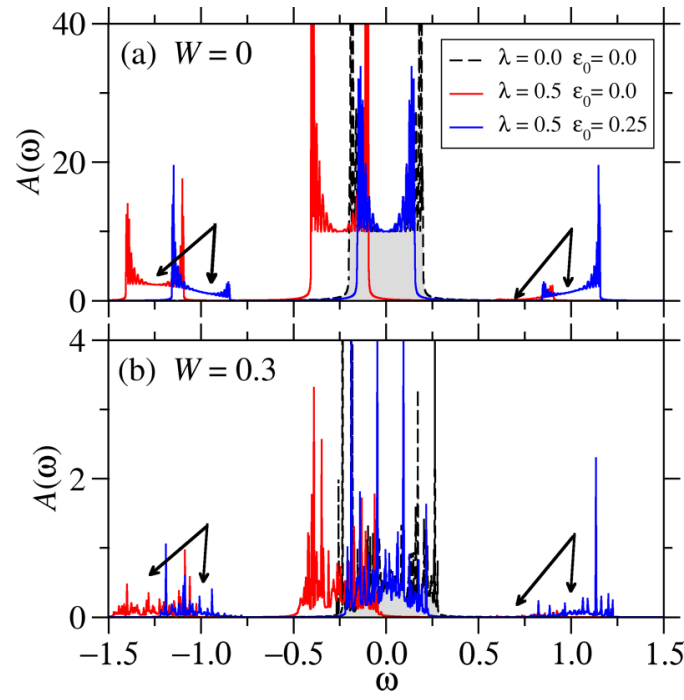

FIG. 3. Spectral function $A(\omega)$ at $k_{B} T=0.1$ for a MNW symmetrically coupled to leads $\left(\Gamma_{L}=\Gamma_{R}=0.2\right)$ with (a) no disorder and (b) random on-site energies $(W=0.3)$, averaged over 100 realizations. The number of molecules is $N=20$. Blue and red solid lines correspond to finite electron-vibron coupling $(\lambda=0.5)$ when $\varepsilon_{0}=0.25$ and 0 , respectively. Black dashed lines show the spectral density of the noninteracting wire $(\lambda=0)$ when $\varepsilon_{0}=0$ (the area under these two curves has been shaded to allow easier identification). For clarity, the error bars of the averages in panel (b) are not shown. The arrows point at the first-order side bands of the interacting systems.

Following the reasoning from Ref. [31], the sum $J_{\Delta E}(\Delta T) \equiv$ $J_{Q}^{L}(\Delta T)+J_{Q}^{R}(\Delta T)=J_{Q}^{L}(\Delta T)+J_{Q}^{L}(-\Delta T)$ equals the rate of energy generated inside the MNW. In the following sections, the electric and heat currents will be expressed in units of $e \omega_{0} / 2 \pi$ and $\omega_{0}^{2} / 2 \pi$, respectively. The superscript $L$ in Eq. (6b) will be removed unless stated otherwise.

\section{SPECTRAL FUNCTION}

In this section, we present and discuss the salient features of the spectral function $A(\omega)$. This quantity provides information of the energy spectrum of the elementary excitations in the system. For concreteness, we restrict ourselves to the equilibrium regime by setting $\mu_{L}=\mu_{R}=0$ and $\Delta T=0$ throughout this section. To gain insight into the effect of the electron-vibron interaction we compare the numerically calculated spectral density in the noninteracting $\operatorname{MNW}(\lambda=0)$ with a wire where the electron-vibron coupling strength is finite $(\lambda=0.5)$. Energies are expressed in units of the vibron energy $\omega_{0}$ in what follows (recall that we set $\hbar=1$ ). As already mentioned, the coupling to the leads is taken as symmetric with $\Gamma_{1,1}^{L}=\Gamma_{N, N}^{R}=0.2\left(\Gamma_{i j}^{\alpha}=0\right.$ otherwise $)$, the bare intermolecular hopping energy is $J=0.1$ and $N=20$.

Figure 3(a) shows the spectral density of a uniform MNW ( $W=0$ ) with constant on-site energy $\varepsilon_{0}=0.25$ (blue solid line) and $\varepsilon_{0}=0$ (red solid line), which corresponds to renormalized energies $\tilde{\varepsilon}_{0}=0$ and $\tilde{\varepsilon}_{0}=-0.25$ when $\lambda=0.5$, respectively. The temperature of the system is $k_{B} T=0.1$. It is important to stress that we are assuming that the system is gated 
so the energy level $\varepsilon_{0}$ can be set at the chemical potential of the contacts at equilibrium $\mu$ [32]. In addition, the Huang-Rhys factor $g=\lambda^{2} / \omega_{0}^{2}$ turns out to be $g=0.25$, which falls in the typical range of parameters of organic semiconductors [33]. Results are compared to the noninteracting case $(\lambda=0$, black dashed line) when $\varepsilon_{0}=0$, whose spectral density displays the expected $U$-shaped profile of width $4 \widetilde{J}$ corresponding to a one-dimensional lattice with dressed intermolecular hopping energy $\widetilde{J}$. When the electron-vibron interaction is turned on (red solid line), the zero-vibron band of the spectral density is red-shifted with regard to the noninteracting case, according to the renormalization of the on-site energy $\tilde{\varepsilon}_{0}=$ $\varepsilon_{0}-\lambda^{2}=-0.25$ associated to the deformation of the lattice around the tunneling electron [34]. Furthermore, the finite electron-vibron interaction leads to the formation of side bands centered at energies $\tilde{\varepsilon}_{0}-1$ and $\tilde{\varepsilon}_{0}+1$, which correspond to emission or absorption of vibrational energy, respectively. As the thermal energy is small compared to the vibrational energy $\left(k_{B} T \ll \omega_{0}\right)$, the latter contribution is weak, which leads to an asymmetric spectral density $A(\omega)$ and therefore to a broken particle-hole symmetry in the case $\widetilde{\varepsilon}_{0} \neq 0$. For the case of a renormalized energy of $\widetilde{\varepsilon}_{0}=0$ (blue solid line), the particle-hole symmetry is established and the side bands are symmetric. It has to be noted that the sum rule $\int \mathrm{d} \omega \mathcal{A}(\omega)=2 \pi$ still holds, so that the additional contribution due to the side channels are compensated by decreasing the height of the zero-vibron band with respect to the noninteracting case, as seen when comparing the blue solid line with the black dashed line [35].

In summary, the effect of the electron-vibron interaction on the spectral density of a uniform MNW is similar to the case of a single molecule [35]. The most significant difference is the level splitting to form a band due to the intermolecular coupling and the resulting narrowing arising from the renormalization of the intermolecular hopping energy $\widetilde{J}$ with respect to the noninteracting case $J$.

We now introduce strong static disorder with magnitude $W=0.3$, which is of the order of the MNW bandwidth. This value is similar to those considered by Ciuchi and Fratini to discuss the temperature dependence of the mobility in rubrene organic field-effect transistors [36]. It implies that the localization length in the noninteracting lattice is smaller than the system size (strong disorder limit) since it is determined from the ratio between the magnitude of disorder and the bandwidth. In other words, this is a key parameter to elucidate the importance of disorder because the larger the ratio $W / J$, the smaller the localization length [37]. When $W / J$ is of the order of unity, the electron becomes mainly localized at a single molecule. Figure 3(b) shows the resulting spectral function when the other parameters are the same as in Fig. 3(a). The displayed values were calculated by averaging over 100 realizations. As expected, the obtained spectral densities show a much more complicated structure but the sum-rule $\int A(\omega) \mathrm{d} \omega=2 \pi$ remains valid, as the distribution of levels becomes random after introducing disorder. The effect of the electron-vibron interaction on the spectral function does not seem to differ much from the case without disorder. In particular, no midgap channels induced by disorder were found, in contrast to short molecular systems with a single defect [38].

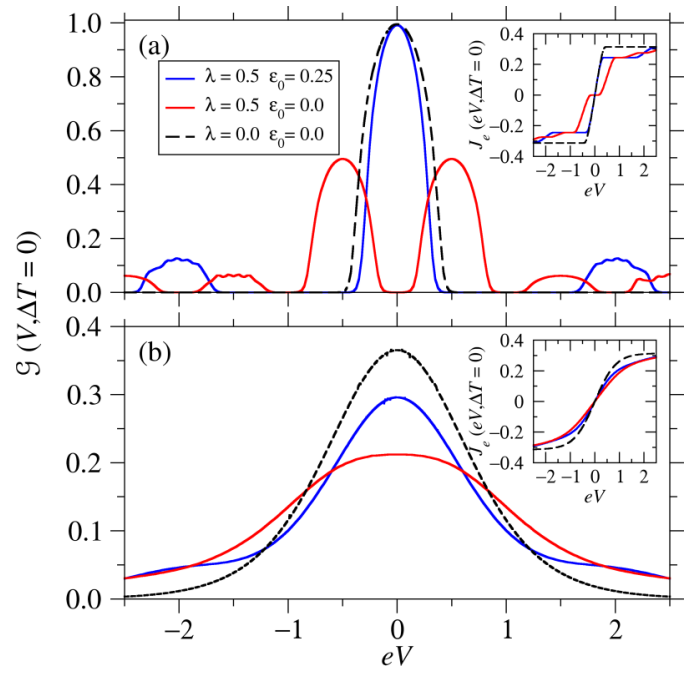

FIG. 4. Differential electric conductance $\mathcal{G}(V, \Delta T=0)=$ $d J_{e} /\left.d V\right|_{\Delta T=0}$ as a function of $e V$ for a MNW without disorder at (a) $k_{B} T=0.01$ and (b) $k_{B} T=0.2$. Blue and red solid lines correspond to finite electron-vibron coupling $(\lambda=0.5)$ when $\varepsilon_{0}=0.25$ and 0 . Black dashed lines show the results for the noninteracting MNW $(\lambda=0)$ when $\varepsilon_{0}=0$. The insets show the corresponding electric currents $J_{e}(e V, \Delta T=0)$.

\section{VOLTAGE-DRIVEN ELECTRIC TRANSPORT}

We now turn to the impact of the electron-vibron interaction on the electric response of the MNW out of equilibrium. To this end, we calculate the nonlinear dependence of the electric current given by Eq. (6a) on the source-drain voltage $V$. As the charge transport across the wire is strongly dominated by resonant tunneling processes, the electric current and the differential conductance $\mathcal{G}(V, \Delta T=0)=d J_{e} /\left.d V\right|_{\Delta T=0}$ give a good insight into the complex nonlinear transmission function of the system. We also calculate the heat current $J_{Q}$ from Eq. (6b). We first discuss the simpler case of the MNW without disorder $(W=0)$ and later compare it to the electric response of the system subjected to disorder ( $W=0.3)$. In both cases, the electric response is computed for a MNW with $(\lambda=0.5)$ and without $(\lambda=0)$ electron-vibron interaction. The rest of parameters is the same as in Fig. 3 .

\section{A. Uniform molecular nanowires}

Figure 4 shows the nonlinear conductance at (a) a low temperature of $k_{B} T=0.01$ and (b) an intermediate temperature of $k_{B} T=0.2$ as a function of $e V$ for a MNW without disorder. The overall shape of the low temperature conductance of the different cases in Fig. 4 follows the general trends of the spectral function shown in Fig. 3(a). This can be easily explained by the close relation between the conductance and the electronic transmission properties through the chain, with peaks corresponding to the resonant transmission channels shown by the spectral function. Notice the smearing out of the conductance due to the finite temperature and the symmetry of $\mathcal{G}(V, \Delta T=0)$ and $J_{e}(e V, \Delta T=0)$ against $\pm e V$. This is related to the upward and downward shift of the chemical potential of the left $(+e V / 2)$ and right $(-e V / 2)$ leads produced by the applied bias. Thus a finite contribution of 
$\mathcal{G}(V, \Delta T=0)$ will correspond to energies $\omega= \pm e V / 2$ where the spectral density is nonzero.

In the case without electron-vibron interaction shown in Fig. 4(a) (black dashed line), the conductance in the uniform MNW only exhibits finite values within the band, as expected. For systems with finite electron-vibron interaction $(\lambda=0.5)$, the differential electric conductance of a MNW with a renormalized on-site energy $\widetilde{\varepsilon}_{0}=0$ (blue solid line) resembles the case without interaction, with a maximum at $e V=0$ corresponding to the zero-vibron peak. The width of the peak is narrower due to the reduced hopping energy $\widetilde{J}<J$. Additionally, the differential conductance also shows side peaks at about $e V= \pm 2$, i.e., $\omega= \pm 1$, which match the transmission channels created by the absorption and emission of vibrons. When the renormalized on-site energy is nonzero (see red solid line, corresponding to $\widetilde{\varepsilon}_{0}=-0.25$ ), the central maximum disappears and two zero-vibron peaks at $e V= \pm 0.5$ arise instead. This is due to the fact that transmission channels are open only for either holes or electrons but not for both simultaneously. In addition, the red solid line shows two more side peaks at $e V= \pm 1.5$ and $e V= \pm 2.5$, the former matching the transmission channel at $\widetilde{\varepsilon}_{0}+1=0.75$ and the latter $\widetilde{\varepsilon}_{0}-1=-1.25$. Interestingly, the weight of the local maxima at $e V= \pm 1.5$ is very large in comparison to the spectral function depicted in Fig. 3. The inset in Fig. 4(a) shows the electric current $J_{e}(V, \Delta T=0)$ corresponding to the conductance of the main panel. $J_{e}(V, \Delta T=0)$ clearly displays the same saturation value independent of $\lambda$, which indicates that we do not induce any real scattering with the electron-vibron interaction and which corresponds to the conservation of the spectral sum-rule discussed in Sec. III. Figure 4(b) displays the conductance at a higher temperature $k_{B} T=0.2$. The different local maxima seen in Fig. 4(a) reduce to a single wide peak due to the thermal smearing out of the Fermi-Dirac distribution in the leads. Also here the saturation values of the electric current with and without electron-vibron interaction are the same.

The MNW presents metallic or semiconducting transport properties, shown in Fig. 4, according to the alignment of the states with respect to the chemical potential. When the center of the band of states matches the chemical potential in equilibrium $\mu=0$, the MNW is metallic and the currentvoltage characteristics is linear around $e V=0$. This is the case of the noninteracting MNW when $\varepsilon_{0}=0$ [black dashed line in Figs. 3 and 4(a)], as seen in Fig. 5(a). The occurrence of a finite electron-vibron interaction shifts the band of states and opens a gap, as depicted in Fig. 5(b). Consequently, the MNW becomes semiconducting. This is in agreement with the observation of a zero differential electric conductance at $e V=0$ shown in Fig. 4(a) (red solid line). The small gap is not observed at high temperature, as expected [see Fig. 4(b)]. Similar comments can be done regarding the side bands of the spectral function that also reveal themselves in the differential electric conductance curves (not shown in Fig. 5 for the sake of clarity).

Figure 6 shows the heat current $J_{Q}(V)$ as a function of $e V$ at low and intermediate temperatures as in Fig. 4. Unlike the electric current, the heat current does not saturate at high $e V$ due to Joule heating. The observed linear behavior of $J_{Q}(V, \Delta T=0)$ at high voltage manifests itself
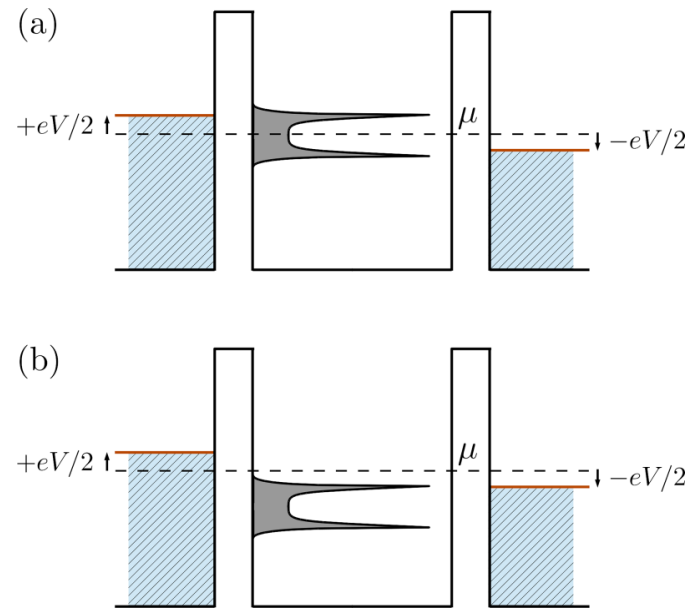

FIG. 5. Level alignment around the chemical potential $\mu$ when the MNW is subject to an applied voltage. (a) When the band of states is located symmetrically about $\mu$ the MNW presents metallic behavior [solid blue and black dashed lines in Figs. 3 and 4(a)]. (b) A finite and large value of the electron-vibron interaction shifts downward the band of states and a gap opens [red solid line in Fig. 4(a)].

in the saturation of the differential electrothermal conductance $\mathcal{M}(V, \Delta T=0)=d J_{Q} /\left.d V\right|_{\Delta T=0}$ (shown in the insets). The electron transmission properties can be depicted in the nonlinear progression of the differential electrothermal conductance $\mathcal{M}$, where we see strong deviations compared to the differential electric conductance $\mathcal{G}$ due to the Joule heating $\mu_{L} J_{e} / e$ and the weighting by the tunneling electron energy $\omega$ in Eq. (6b). One should note the strong resemblance of the $J_{Q}(V, \Delta T=0)$ curves without electron-vibron interaction (black dashed lines) and the corresponding curve with the

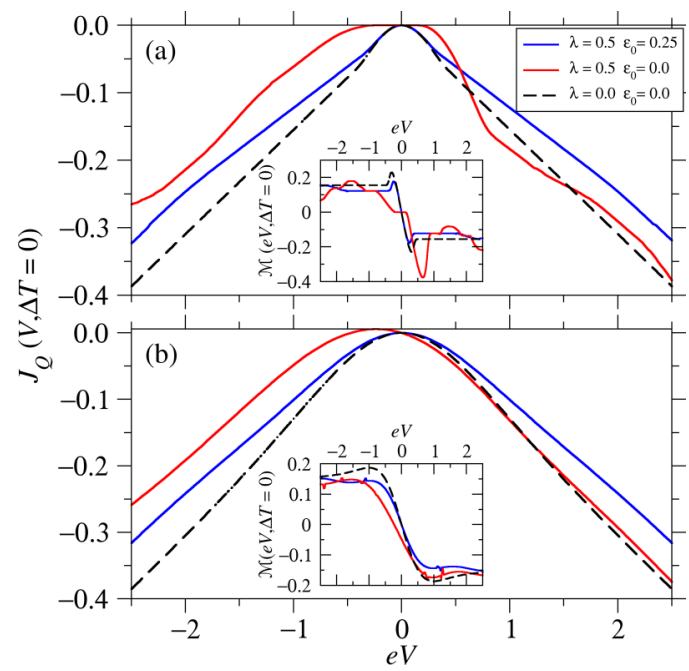

FIG. 6. Heat current $J_{Q}(V, \Delta T=0)$ as a function of $e V$ for MNWs without disorder as in the case of Fig. 4 at (a) $k_{B} T=0.01$ and (b) $k_{B} T=0.2$. Blue and red solid lines correspond to finite electron-vibron coupling $(\lambda=0.5)$ when $\varepsilon_{0}=0.25$ and 0 . Black dashed lines show the results for the noninteracting wire $(\lambda=0)$ when $\varepsilon_{0}=0$. The insets display the corresponding differential electrothermal conductance $\mathcal{M}(V, \Delta T=0)=d J_{Q} /\left.d V\right|_{\Delta T=0}$. 


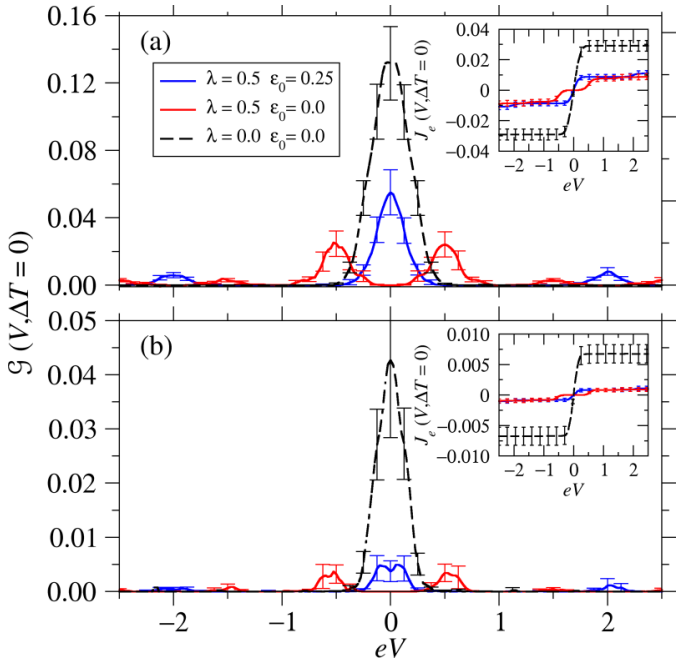

FIG. 7. Differential electric conductance $\mathcal{G}(V, \Delta T=0)=$ $d J_{e} /\left.d V\right|_{\Delta T=0}$ at $k_{B} T=0.01$ as a function of $e V$ of MNWs of length (a) $N=20$ and (b) 40 subjected to disorder ( $W=0.3$ ). Results were averaged over 100 realizations. The other parameters are the same as in Fig. 4. Blue and red solid lines correspond to finite electron-vibron coupling $(\lambda=0.5)$ when $\varepsilon_{0}=0.25$ and 0 . Black dashed lines show the results for the noninteracting wire $(\lambda=0)$ when $\varepsilon_{0}=0$. The inset shows the corresponding electric current $J_{e}(e V, \Delta T=0)$.

coupling switched on for the same bare on-site energy $\varepsilon_{0}=0$ (red solid lines) for $e V>0$. This can be explained by the small contribution of thermally generated vibrons at low background temperatures, so that the total energy is nearly conserved when switching on the electron-vibron interaction. Therefore, when the electric bias is high enough and all vibronic side bands are open for transmission, the total energy transferred to the system is approximately conserved after switching on the interaction. On the contrary, this conservation does not hold for an electric bias such $e V<0$ due to the breaking of the electron-hole symmetry (asymmetry of the transmission about $\omega=0$ ) by switching on the interaction, as discussed in the previous section. Unlike the symmetric electric current $J_{e}(V)$, this asymmetry transmission can be seen in the heat current from the left lead to the MNW (6b). On the other hand, the blue solid lines, which correspond to a renormalized on-site energy $\widetilde{\varepsilon}_{0}=0$ and therefore a symmetric transmission, also shows a heat current symmetric about $e V=0$.

\section{B. Disordered molecular nanowires}

Figure 7(a) shows the differential electric conductance $\mathcal{G}(V, \Delta T=0)$ of disordered MNWs with $W=0.3$ at $k_{B} T=$ 0.01 , averaged over 100 realizations, and the other parameters as in Fig. 4. The main features of the curves resemble those without disorder, the main difference being the occurrence of sharper peaks and a strong reduction of the maximum differential electric conductance. The conductance is reduced by a factor of $\sim 8$ for the chain without electron-vibron interaction, and up to $\sim 15$ for the interacting case. When temperature is increased to $k_{B} T=0.2$ the peaks of the differential conductance become broader and even merge into

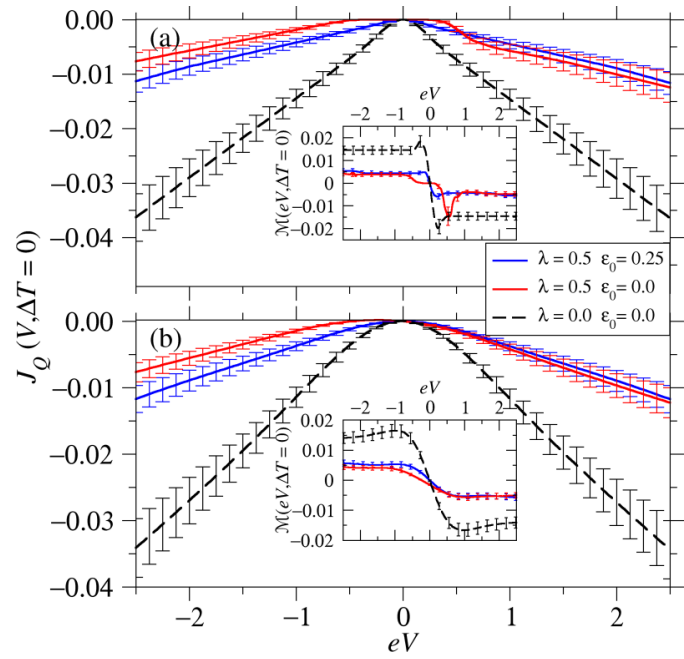

FIG. 8. Heat current $J_{Q}(V, \Delta T=0)$ as a function of $e V$ of MNWs with disorder $(W=0.3)$ averaged over 100 realisations at (a) $k_{B} T=0.01$ and (b) $k_{B} T=0.2$. The other parameters are the same as in Fig. 6. Blue and red solid lines correspond to finite electron-vibron coupling $(\lambda=0.5)$ when $\varepsilon_{0}=0.25$ and 0 . Black dashed lines show the results for the noninteracting wire $(\lambda=0)$ when $\varepsilon_{0}=0$. The insets show the corresponding differential electrothermal conductance $\mathcal{M}(V, \Delta T=0)=d J_{Q} /\left.d V\right|_{\Delta T=0}$.

a single one in the case $\lambda=0.5$ and $\varepsilon_{0}=0$ (not shown) as for the ordered MNW in Fig. 4(b).

In addition, the differential electric conductance is further decreased if the length of the MNW increases from $N=20$ to 40, as displayed in Fig. 7(b). This behavior is in contrast to the uniform MNW, where the conductance is preserved against the system length. It is worth noting the stronger reduction in the MNW with finite electron-vibron interaction when doubling the length, compared to the case without interaction. Such a reduction can be traced back to the Anderson localization of electron states $[1,2,10]$. In general, the magnitude of disorder has to be compared with the hopping energy or, in other words, with the bandwidth of the uniform system. The larger the ratio between them, the smaller the localization length. From this reasoning, the effects of disorder should be more important on increasing temperature when the electron-vibron coupling is finite since the exponential suppression of tunneling reduces the dressed intermolecular hopping energy $\widetilde{J}$. The expected reduction of the localization length is hinted from the comparison of Figs. 4 and 7.

For completeness, Fig. 8 shows the much reduced heat current $J_{Q}(V, \Delta T)$ in disordered MNWs with $W=0.3$ as compared to Fig. 6. The influence of disorder agrees well with the observed electric current, supporting the idea of an increased localization in the case of finite electron-vibron interaction that influences both charge and heat transport alike.

\section{TEMPERATURE-DRIVEN ELECTRIC TRANSPORT}

We now investigate the electric transport through MNWs in response to a temperature difference $\Delta T$ only. We are assuming a symmetrically biased system with $T_{L}=T-\Delta T / 2$ and $T_{R}=T+\Delta T / 2$, therefore keeping the average temperature 


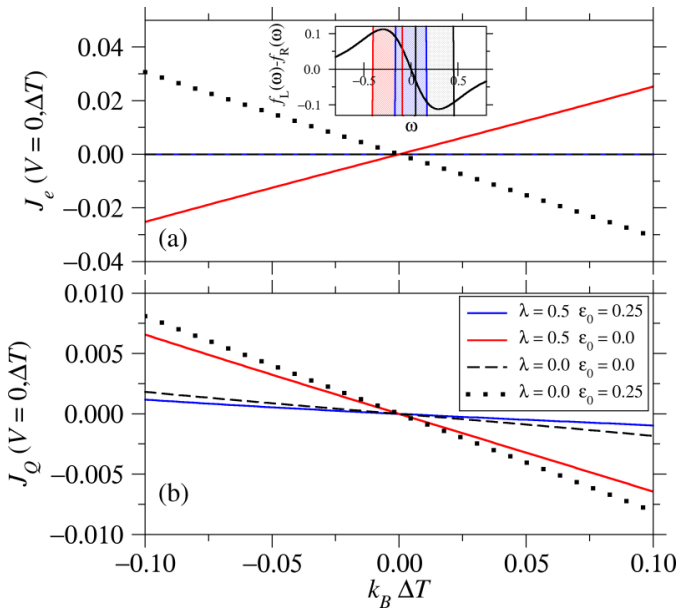

FIG. 9. Temperature-driven (a) electric current $J_{e}$ and (b) heat current $J_{Q}$ as a function of the temperature difference $\Delta T$ for MNWs without disorder $(W=0)$ at $k_{B} T=0.2$. Blue and red solid lines correspond to finite electron-vibron coupling $(\lambda=0.5)$ when $\varepsilon_{0}=0.25$ and $\varepsilon_{0}=0$. Black dashed (superimposed on the blue solid line) and dotted lines show the results for the noninteracting wire $(\lambda=0)$ when $\varepsilon_{0}=0$ and 0.25 , respectively. Inset represents $f_{L}(\omega)-f_{R}(\omega)$ as a function of the frequency $\omega$ together with a colored area representing the frequency region where the spectral density is finite for every parameter set.

$\left(T_{L}+T_{R}\right) / 2$ at a defined value $T$. In order to ensure that each site-dependent $T_{i}>0$, the maximum $\Delta T_{\max }$ is fixed at $\Delta T_{\max }= \pm T / 2$ so that the leads do not deviate more then $25 \%$ from their initial temperature. In this section, the bias voltage is absent $(V=0)$ and charge flows through the MNW only due to the temperature difference between the leads. As in Sec. IV, we first discuss the electric and heat currents in uniform MNWs $(W=0)$ and later we consider random on-site energies with $W=0.3$. The other parameters of the system are taken the same as in Sec. IV, with the additional case of a noninteracting MNW with on-site energy $\varepsilon_{0}=0.25$.

\section{A. Uniform molecular nanowires}

Figure 9(a) shows the electric current $J_{e}(V=0, \Delta T)$ at $k_{B} T=0.2$ as a function of $\Delta T$ for a $\mathrm{MNW}$ without disorder. The blue solid line $\left(\lambda=0.5\right.$ and $\left.\widetilde{\varepsilon}_{0}=0\right)$ and the black dashed line $\left(\lambda=0\right.$ and $\left.\varepsilon_{0}=0\right)$, which represent systems with symmetric transmission around the Fermi energy $\mu_{L}=$ $\mu_{R}=0$, do not exhibit any thermoelectric current at all. This is due to the fact that, when a thermal bias is present, all electrons tunneling from the hot lead to the unoccupied states of the cold lead are compensated by holes tunneling from the unoccupied states to the still occupied state of the cold lead due to the electron-hole symmetry. If this symmetry is broken, as in the case of the red solid line $\left(\lambda=0.5\right.$ and $\left.\widetilde{\varepsilon}_{0}=-0.25\right)$ and the blue dotted line $\left(\lambda=0\right.$ and $\left.\varepsilon_{0}=0.25\right)$, the system exhibits a finite thermally-driven electron or hole current. The inset of Fig. 9(a) links these results to those related to the spectral density in Sec. III. According to Eq. (6a) the most relevant contribution to $J_{e}$ can be approximated by the frequency integration of the magnitude $\left[f_{L}(\omega)-f_{R}(\omega)\right] \mathcal{A}(\omega)$. The inset plots the odd function $f_{L}(\omega)-f_{R}(\omega)$ together with colored areas representing the frequency range where the spectral densities are relevant. As seen in Fig. 3(a), the spectral density is centered at $\omega=0$ when $\widetilde{\varepsilon}_{0}=0.0$ (blue area) and it is shifted to lower (higher) frequencies when $\widetilde{\varepsilon}_{0}<0.0\left(\widetilde{\varepsilon}_{0}>0.0\right)$ (red and grey areas). Thus, it is clear that the signs and symmetries of both factors justify the values of $J_{e}$ presented in the main plot. Unlike the voltage-driven case, this current is nearly linear since the temperature difference is not high enough to reach a nonlinear regime.

Figure 9(b) displays the heat current $J_{Q}(V=0, \Delta T)$ at $k_{B} T=0.2$ as a function of $\Delta T$. Unlike the electric current $J_{e}(V=0, \Delta T)$, also the blue solid line $\left(\lambda=0.5\right.$ and $\left.\widetilde{\varepsilon}_{0}=0\right)$ and the black dashed line $\left(\lambda=0\right.$ and $\left.\varepsilon_{0}=0\right)$ exhibit finite values due to weighting of the tunneling particles with their respective energies and the asymmetry of the heat currents from the left and the right leads $\left(J_{Q}^{L} \neq J_{Q}^{R}\right)$. As the majority of the carriers are transmitted through the zero-vibron channel, the transmitted energy and therefore the heat current is rather low compared to the other two cases, whose main transmission channels are centered at a finite energy. A small influence of the electron-vibron interaction can be seen as a slight asymmetry of the absolute value of the thermal current $\left|J_{Q}(\Delta T)\right| \neq\left|J_{Q}(-\Delta T)\right|$ for the cases with finite $\lambda$. Such an effect can also be depicted for disordered MNWs in the next section and it shall be discussed on that behalf.

\section{B. Disordered molecular nanowires}

Figure 10(a) shows the average temperature-driven $J_{e}(V=$ $0, \Delta T)$ as a function of $\Delta T$ for the same systems shown in Fig. 9 but with disorder $W=0.3$. As in the case of voltagedriven transport discussed in Sec. IV, disorder strongly alters the electric response of the MNW. We observe that it affects the interacting MNW more significantly than the noninteracting one. This can be explained by the same reasoning introduced in Sec. IV.

Figure 10(b) displays the temperature-driven $J_{Q}(V=$ $0, \Delta T$ ) for the same parameters as in Fig. 10(a). One can see a strong nonlinear curve progression for MNWs with finite coupling. More importantly, the interacting system (blue solid line) actually exhibits a stronger heat current in comparison with the non-interacting one (black dashed line) for $\Delta T<0$. As can be concluded from the effect of disorder seen in the electric current in Fig. 10(a), this can not be related to the electric response of the system. Therefore it must arise from the heat transport properties. Due to the symmetry of the system at $\Delta T=0, J_{Q}^{L}(-\Delta T)=J_{Q}^{R}(\Delta T)$, the rate of energy generation inside the system is $J_{\Delta E}(\Delta T) \equiv J_{Q}^{L}(\Delta T)+$ $J_{Q}^{L}(-\Delta T)$. Thus the asymmetry between the $J_{Q}^{L}(-\Delta T)$ and $J_{Q}^{L}(\Delta T)$ must be explained by a heat generation process inside the system. As this process is only noticeable in interacting MNWs, it is reasonable to assume that it is due to the thermal generation of vibrons. At $k_{B} T=0.2$ the amount of thermally generated vibrons is very small, which is why the asymmetry is much less visible in case of no disorder in Fig. 9(b). However, when actually calculating the heat generation rate $J_{\Delta E}$, as depicted in Fig. 10(c) for the case of $\varepsilon_{0}=0$, in good approximation, one gets the same values for the ordered (magenta curve) and the disordered MNW (red curve). This justifies the 


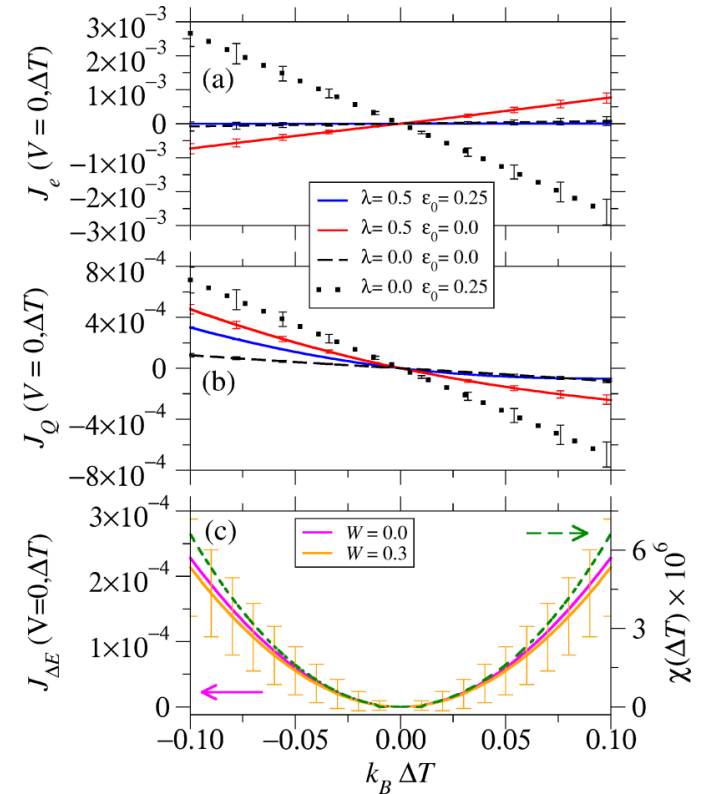

FIG. 10. Temperature-driven (a) electric current $J_{e}$ and (b) heat current $J_{Q}$ as a function of the temperature difference $\Delta T$ for MNWs with disorder $(W=0.3)$ at $k_{B} T=0.2$. Blue and red solid lines correspond to finite electron-vibron coupling $(\lambda=0.5)$ when $\varepsilon_{0}=0.25$ and 0 . Black dashed and dotted lines show the results for the noninteracting wire $(\lambda=0)$ when $\varepsilon_{0}=0$ and 0.25 , respectively. (c) displays the heat generation inside the MNW $J_{\Delta E}$ as a function of $\Delta T$ at $k_{B} T=0.2$ for an $\varepsilon_{0}=0.25$ in the case without disorder (magenta solid line) and with disorder (orange solid line) calculated from Fig. 9(b) and 10(b), respectively. The green dashed line represents the occupation number of thermally generated vibrons times the thermoelectric current $\chi(\Delta T)$ in arbitrary units as a function of $\Delta T$.

explanation of an effect governed by the vibronic subsystem. In addition, the effect of heat generation gets further support when looking at the green curve in Fig. 10(c) that shows the magnitude $\chi(\Delta T)=J_{e}(V=0, \Delta T) \sum_{i=1}^{N} n_{i}\left(T_{i}\right)$. It represents the occupation number of thermally generated vibrons inside the system times the electric current and, consequently, it provides a rough estimation of the number of electrons to which the vibrons can couple. As the green curve clearly is proportional to the heat generation rate $J_{\Delta E}$, one can safely conclude that the additional heat is transferred from the heat baths to the MNW.

\section{CONCLUSIONS}

In conclusion, we have studied the nonequilibrium transport properties of a disordered MNW. The wire is regarded as a quasi-one-dimensional organic crystal of random single-level molecules, connected in series to two leads. We have also assumed that the electron interacts with local vibrational modes in the molecules and investigated the effects of the interaction on the electric and heat currents in response to either an applied electric bias or temperature gradient established in the system. The original many-body problem has been turned into an effective one-body problem by the polaron (Lang-Firsov) transformation.
We have considered the regime of strong disorder, for which the localization length in the noninteracting $\operatorname{MNW}(\lambda=0)$ is smaller than the system size. In addition, we have taken $k_{B} T \ll \omega_{0}$ in our simulations and consequently the scattering is mainly dominated by the interaction with the disordered lattice. This is supported by the fact that the voltage-driven electric current $J_{e}$ in uniform MNWs $(W=0)$ is independent of the system length and its saturation value is the same for both noninteracting and interacting cases. In general, voltage- and temperature-driven electric currents present a similar decrease due to disorder or electron-vibron coupling. Remarkably, we have found that the electron-vibron interaction enhances the effects of the disorder on the electric and heat currents. This important result can be understood as follows. The intermolecular hopping energy $\widetilde{J}_{i}$ in the interacting MNW is smaller than the bare $J$ due to the occurrence of the exponential suppression of tunneling [25]. Therefore disorder is effectively stronger when the interaction is switched on because the ratio between the magnitude of disorder and the bandwidth increases.

Regarding the temperature-driven transport, we have numerically found an almost linear dependence of the electric and heat currents on the temperature difference. In MNWs with preserved electron-hole symmetry, the electric current vanishes even if the electron-vibron interaction is taken into account. On the contrary, the heat current is always nonzero and enhanced due to the interaction, although to a small extent. Most importantly, the effects of disorder are more pronounced in the interacting MNW. Disorder reveals itself by a slight deviation of the temperature-driven heat current curves from linearity. In addition, the temperature-driven heat current is less sensitive to the increase of the magnitude of disorder or the electron-vibron coupling than the electric current.

In order to get a clear connection with experiments, a look at the magnitudes of interest in physical units is in order. Our energy unit thus far has been the vibron frequency $\omega_{0}$ so the following numbers strongly depend on the details of our molecular bridge (see Ref. [39] for a review on this topic). By taking a reference value of $\omega_{0}=150 \mathrm{meV}$, our study focuses on a physical scenario where the conducting molecular level is $\varepsilon_{0} \sim 40-80 \mathrm{meV}$ and the electron coupling with the vibrons and the leads are $\lambda=10 \mathrm{fs}$ and $\Gamma^{L / R}=30 \mathrm{meV}$, respectively. These parameters are consistent with those found by experiments [32] with charge and heat currents of the order of $J_{e} \sim$ $10-100 \mathrm{nA}$ and $J_{Q} \sim 1-10 \mathrm{nW}$ at achievable temperatures of $T \sim 15-350 \mathrm{~K}$.

Although this work is focused on the study to MNWs, our results can be extended to arrays of quantum dots as well. These arrays can be realized in electron gases with superposed mesh gates. Beside their interest in fundamental research, they are regarded as good candidates for building quantum simulators [40-42]. Unavoidable imperfections introduced during the fabrication process might have an impact on charge and energy transport when electrons are coupled to bosonic degrees of freedom. Our results shed light on the influence of disorder on the performance of quantum simulators based on arrays of quantum dots. 


\section{ACKNOWLEDGMENTS}

The authors are grateful to D. Sánchez, M. A. Sierra, and C. Álvarez for helpful discussions. F. D-A. thanks the Theoretical Physics Group of the University of Warwick for the warm hospitality. Work at Madrid has been supported by MINECO under Grants No. MAT2013-46308 and No. MAT2016-75955. UK research data statement: all data accompanying this publication are directly available within the publication.

\section{APPENDIX A: LANG-FIRSOV POLARON TRANSFORMATION}

Starting from Eqs. (1)-(3), we apply the polaron (Lang-Firsov [29]) nonperturbative canonical transformation $\widetilde{H}=e^{S} H e^{-S}$, where the operator $S$ is defined as $S=$ $\left(\lambda / \omega_{0}\right) \sum_{i}\left(a_{i}^{\dagger}-a_{i}\right) c_{i}^{\dagger} c_{i}$ [29]. This transformation yields the following transformed Hamiltonian:

$$
\begin{aligned}
\widetilde{H}= & \sum_{i=1}^{N} \widetilde{\varepsilon}_{i} c_{i}^{\dagger} c_{i}-J \sum_{i=1}^{N-1}\left(X_{i}^{\dagger} X_{i+1} c_{i}^{\dagger} c_{i+1}+\text { H.c }\right) \\
& +\omega_{0} \sum_{i=1}^{N} a_{i}^{\dagger} a_{i}+\sum_{\alpha k i}\left(V_{\alpha k i} X_{i} d_{\alpha k}^{\dagger} c_{i}+\text { H.c }\right),
\end{aligned}
$$

where $\widetilde{\varepsilon}_{i}=\varepsilon_{i}-\lambda^{2} / \omega_{0}$ is the renormalized energy level of the molecule $i$. After the transformation, a new operator arises, $X_{i}=\exp \left[-\left(\lambda / \omega_{0}\right)\left(a_{i}^{\dagger}-a_{i}\right)\right]$, named the displacement operator. The canonical transformation is exact but it does not diagonalize the Hamiltonian. In other words, $\widetilde{H}$ still contains products of boson and fermion operators. When the coupling to the leads is weak $\left(\left|V_{\alpha k i}\right|<\lambda\right)$, it is reasonable to replace the displacement operator $X_{i}$ by its thermal expectation value evaluated in equilibrium $\left\langle X_{i}\right\rangle=\exp \left[-\xi_{i}\left(T_{i}\right) / 2\right]$ [35,43], where $\xi_{i}\left(T_{i}\right)=g\left(2 n_{i}+1\right), n_{i}=1 /\left[\exp \left(\omega_{0} / k_{B} T_{i}\right)-1\right]$ and the Huang-Rhys factor is $g=\lambda^{2} / \omega_{0}^{2}$. By way of this procedure one can deal with an effective one-body problem according to Eq. (4).

\section{APPENDIX B: NONEQUILIBRIUM GREEN'S FUNCTIONS}

The various Green's functions of the system described by Eq. (4) are lengthy but straightforward to calculate numerically with the help of the Keldysh nonequilibrium Green's function formalism [30,39]. First, since we replaced the displacement operator $X_{i}$ by its thermal expectation value, the Green's functions are then factored out. The greater Green's function can be cast in the form

$$
G_{i j}^{>}(t)=\widetilde{G}_{i j}^{>}(t)\left\langle X_{i}(t) X_{j}^{\dagger}(0)\right\rangle,
$$

where $\widetilde{G}_{i j}^{>}(t)$ denotes the so-called dressed greater Green's function. $i \widetilde{G}_{i j}^{>}(t)$ is the correlation function of a hole dressed by vibrons. Concerning the vibron part, we may encounter two different cases. If $i \neq j$ then $\left\langle X_{i}(t) X_{j}^{\dagger}(0)\right\rangle=$ $\exp \left[-\xi_{i}\left(T_{i}\right) / 2-\xi_{j}\left(T_{j}\right) / 2\right]$. When $i=j$ the calculation of the correlation function is more involved but can be performed analytically. The details are presented in Ref. [44] and the final result is

$$
\left\langle X_{i}(t) X_{i}^{\dagger}(0)\right\rangle=\sum_{n=-\infty}^{\infty} L_{n}^{i}\left(T_{i}\right) e^{\mathrm{i} n \omega_{0} t},
$$

where at finite temperature

$$
L_{n}^{i}\left(T_{i}\right)=e^{-\xi_{i}\left(T_{i}\right)+n \omega_{0} / 2 k_{B} T_{i}} I_{n}\left(\sqrt{\xi_{i}^{2}\left(T_{i}\right)-g^{2}}\right),
$$

with $I_{n}(z)$ the modified Bessel function of integer order, and at $T_{i}=0$,

$$
L_{n}^{i}(0)= \begin{cases}e^{-g} g^{n} / n ! & \text { if } n \geqslant 0, \\ 0 & \text { if } n<0 .\end{cases}
$$

Using the vibron mean values given above, the elements of the greater Green's functions, $G^{>}(\omega)$, are given in Fourier space as [35]

$$
\begin{aligned}
& G_{i i}^{>}(\omega)=\sum_{n=-\infty}^{\infty} L_{n}^{i}\left(T_{i}\right) \widetilde{G}_{i i}^{>}\left(\omega-\omega_{0} n\right), \\
& G_{i j}^{>}(\omega)=\left\langle X_{i}\right\rangle\left\langle X_{j}\right\rangle \widetilde{G}_{i j}^{>}(\omega), \quad i \neq j,
\end{aligned}
$$

and similarly for the lesser Green's function, $G^{<}(\omega)$, but replacing $\omega-\omega_{0} n$ by $\omega+\omega_{0} n$ in the summation. The dressed lesser and greater Green's functions can be calculated from the Keldysh equation $\widetilde{G}^{<(>)}(\omega)=\widetilde{G}^{\mathrm{r}}(\omega) \widetilde{\Sigma}^{<(>)}(\omega) \widetilde{G}^{\mathrm{a}}(\omega)$, where the self-energies are given by

$$
\begin{aligned}
& \widetilde{\Sigma}^{<}(\omega)=\mathrm{i}\left[f_{L}^{(e)}(\omega) \widetilde{\Gamma}^{L}+f_{R}^{(e)}(\omega) \widetilde{\Gamma}^{R}\right], \\
& \widetilde{\Sigma}^{>}(\omega)=-\mathrm{i}\left[f_{L}^{(h)}(\omega) \widetilde{\Gamma}^{L}+f_{R}^{(h)}(\omega) \widetilde{\Gamma}^{R}\right],
\end{aligned}
$$

with $f_{\alpha}^{(e)}(\omega)=f_{\alpha}(\omega)$ and $f_{\alpha}^{(h)}(\omega)=1-f_{\alpha}(\omega)$. Here $f_{\alpha}(\omega)=$ $1 /\left\{\exp \left[\left(\omega-\mu_{\alpha}\right) / k_{B} T_{\alpha}\right]+1\right\}$ is the Fermi-Dirac distribution function of the lead $\alpha$. The matrix elements of $\widetilde{\Gamma}^{\alpha}$ in (B6b) are given as $\widetilde{\Gamma}_{i j}^{\alpha}=2 \pi \rho_{\alpha} \widetilde{\widetilde{V}}_{\alpha k i} \widetilde{V}_{\alpha k j}^{*}$, where $\rho_{\alpha}$ is the density of states of the corresponding lead. Notice that we will neglect their $k$ dependence by relying on the wide-band limit approximation and take $\widetilde{\Gamma}^{\alpha}$ matrices as energy-independent magnitudes.

In order to calculate the dressed retarded Green's function $\widetilde{G}^{\mathrm{r}}(\omega)$, the equation-of-motion method is used. We start by calculating the time-derivative of its formal definition $\widetilde{G}_{i j}^{\mathrm{r}}(t)=$ $-\mathrm{i} \theta(t)\left\langle\left\{c_{i}(t), c_{j}^{\dagger}(0)\right\}\right\rangle$, where $t \rightarrow t+\mathrm{i} 0^{+}$, keeping in mind that $\mathrm{i} \delta_{t} c_{i}(t)=\left[c_{i}(t), \widetilde{H}\right]$ where $\widetilde{H}$ is given by Eq. (4) and $\theta(t)$ the Heaviside step function. After Fourier transform, one can write the following system of linear equations to be solved:

$$
\begin{aligned}
\left(\omega-\widetilde{\varepsilon}_{i}\right) \widetilde{G}_{i j}^{\mathrm{r}}(\omega)= & \delta_{i j}-\widetilde{J}_{i} \widetilde{G}_{i+1, j}^{\mathrm{r}}(\omega)-\widetilde{J}_{i-1} \widetilde{G}_{i-1, j}^{\mathrm{r}}(\omega) \\
& +\sum_{l=1}^{N} \widetilde{\Sigma}_{i l}^{\mathrm{r}} \widetilde{G}_{l j}^{\mathrm{r}}(\omega)
\end{aligned}
$$

where

$$
\widetilde{\Sigma}_{i l}^{\mathrm{r}}=\sum_{\alpha k} \frac{\widetilde{V}_{\alpha k i}^{*} \widetilde{V}_{\alpha k l}}{\omega-\varepsilon_{\alpha, k}+\mathrm{i} 0^{+}}
$$

is the retarded self-energy. Within the wide-band approximation this term is written as

$$
\widetilde{\Sigma}_{i l}^{r}(\omega)=\frac{\mathrm{i}}{2}\left\langle X_{i}\right\rangle\left\langle X_{l}\right\rangle\left(\Gamma_{i l}^{L}+\Gamma_{i l}^{R}\right) .
$$


Similarly, to calculate the dressed advanced Green's function $\widetilde{G}_{i j}^{\mathrm{a}}(\omega)$, one can use Eq. (B7) by substituting $\widetilde{\Sigma}_{i l}^{r}(\omega)$ by the following advanced self-energy:

$$
\widetilde{\Sigma}_{i l}^{a}(\omega)=-\frac{\mathrm{i}}{2}\left\langle X_{i}\right\rangle\left\langle X_{l}\right\rangle\left(\Gamma_{i l}^{L}+\Gamma_{i l}^{R}\right) .
$$

$(\mathrm{B} 8 \mathrm{c})$
[1] P. W. Anderson, Phys. Rev. 109, 1492 (1958).

[2] N. F. Mott and W. D. Twose, Adv. Phys. 10, 107 (1961).

[3] M. Kohmoto and B. Sutherland, Phys. Rev. Lett. 56, 2740 (1986).

[4] F. A. B. F. de Moura and M. L. Lyra, Phys. Rev. Lett. 81, 3735 (1998).

[5] V. Bellani, E. Diez, R. Hey, L. Toni, L. Tarricone, G. B. Parravicini, F. Domínguez-Adame, and R. Gómez-Alcalá, Phys. Rev. Lett. 82, 2159 (1999).

[6] A. Rodríguez, V. A. Malyshev, and F. Domínguez-Adame, J. Phys. A: Math. Gen. 33, L161 (2000).

[7] U. Kuhl, F. M. Izrailev, A. A. Krokhin, and H.-J. Stöckmann, Appl. Phys. Lett. 77, 633 (2000).

[8] S. L. A. de Queiroz, Phys. Rev. B 66, 195113 (2002).

[9] A. Rodríguez, A. Chakrabarti, and R. A. Römer, Phys. Rev. B 86, 085119 (2012).

[10] E. Abrahams, P. W. Anderson, D. C. Licciardello, and T. V. Ramakrishnan, Phys. Rev. Lett. 42, 673 (1979).

[11] G. Kopidakis, C. M. Soukoulis, and E. N. Economou, Europhys. Lett. 33, 459 (1996).

[12] F. X. Bronold, A. Alvermann, and H. Fehske, Philos. Mag. 84, 673 (2004).

[13] A. A. Bakulin, R. Lovrincic, X. Yu, O. Selig, H. J. Bakker, Y. L. A. Rezus, P. K. Nayak, A. Fonari, V. Coropceanu, J.-L. Brédas, and D. Cahen, Nat. Commun. 6, 7880 (2015).

[14] A. Erpenbeck, R. Härtle, and M. Thoss, Phys. Rev. B 91, 195418 (2015).

[15] W. Wang, T. Lee, I. Kretzschmar, and M. A. Reed, Nano Lett. 4, 643 (2004).

[16] M. Galperin, M. A. Ratner, and A. Nitzan, Nano Lett. 4, 1605 (2004).

[17] S. Kubatkin, A. Danilov, M. Hjort, J. Cornil, J.-L. Brédas, N. Stuhr-Hansen, P. Hedegård, and T. Bjørnholm, Nature 425, 698 (2003).

[18] J. Lykkebo, A. Gagliardi, A. Pecchia, and G. C. Solomon, ACS Nano 7, 9183 (2013).

[19] S. Fratini, D. Mayou, and S. Ciuchi, Adv. Funct. Mater. 26, 2292 (2016).

[20] G. Nan, X. Yang, L. Wang, Z. Shuai, and Y. Zhao, Phys. Rev. B 79, 115203 (2009).

[21] Y. Wang, J. Zhou, and R. Yang, J. Phys. Chem. C 115, 24418 (2011).
[22] J. L. D’Amato and H. M. Pastawski, Phys. Rev. B 41, 7411 (1990).

[23] T. Grange, Phys. Rev. B 89, 165310 (2014).

[24] H. Haug and A. P. Jauho, Quantum Kinetics in Transport and Optics of Semiconductors (Springer, Berlin, 2007).

[25] W. Rudziński, J. Phys.: Condens. Matter 20, 275214 (2008).

[26] C. Ávarez, F. Domínguez-Adame, P. A. Orellana, and E. Díaz, Phys. Lett. A 379, 1062 (2015).

[27] V. Coropceanu, J. Cornil, D. A. da Silva Filho, Y. Olivier, R. Silbey, and J.-L. Brédas, Chem. Rev. 107, 926 (2007).

[28] T. Holstein, Ann. Phys. 8, 325 (1959).

[29] I. G. Lang and Y. A. Firsov, Zh. Eksp. Teor. Fiz. 43, 1843 (1963) [Sov. Phys. JETP 16, 1301 (1963)].

[30] L. V. Keldysh Zh. Eksp. Teor. Fiz. 47, 1515 (1965) [Sov. Phys. JETP 20, 1018 (1965)].

[31] M. Galperin, A. Nitzan, and M. A. Ratner, Phys. Rev. B 75, 155312 (2007).

[32] M. Poot, E. Osorio, K. O’Neill, J. M. Thijssen, D. Vanmaekelbergh, C. A. van Walree, L. W. Jenneskens, and H. S. J. van der Zant, Nano Lett. 6, 1031 (2006).

[33] N. Ueno, S. Kera, K. Sakamoto, and K. K. Okudaira, Appl. Phys. A 92, 495 (2008)

[34] N. S. Wingreen, K. W. Jacobsen, and J. W. Wilkins, Phys. Rev. Lett. 61, 1396 (1988).

[35] Z.-Z. Chen, R. Lü, and B.-f. Zhu, Phys. Rev. B 71, 165324 (2005).

[36] S. Ciuchi and S. Fratini, Phys. Rev. B 86, 245201 (2012).

[37] F. Domínguez-Adame and V. A. Malyshev, Am. J. Phys. 72, 226 (2004).

[38] D. Nozaki, H. M. Pastawski, and G. Cuniberti, New J. Phys. 12, 063004 (2010).

[39] M. Galperin, M. A. Ratner, and A. Nitzan, J. Phys.: Condens. Matter 19, 103201 (2007).

[40] I. Buluta and F. Nori, Science 326, 108 (2009).

[41] S. J. Chorley, C. G. Smith, F. Pérez-Martínez, J. Prance, P. Atkinson, D. A. Ritchie, and G. A. C. Jones, Microelectron. J. 39, 314 (2008).

[42] J. Gray, A. Bayat, R. K. Puddy, C. G. Smith, and S. Bose, Phys. Rev. B 94, 195136 (2016).

[43] B. H. Wu, J. C. Cao, and C. Timm, Phys. Rev. B 86, 035406 (2012).

[44] G. D. Mahan, Many-Particle Physics (Kluwer Academic, New York, 2000). 